\title{
Hiding in plain sight: On culturally responsive evaluation and LGBT communities of colour Robin Lin Miller
}

Keynote Address for 4th Annual Conference of the Center for Culturally Responsive Evaluation and Assessment

29 September, 2017

Chicago, Illinois

When Stafford ${ }^{1}$ invited me to address you, I felt momentarily unsettled. An interaction I had with a renowned African scholar who visited Michigan State University (MSU) last March speaks to the basis for my unease. I had been asked to host her for a roundtable discussion with students and faculty. A prominent player in the South African movement to protect indigenous knowledge and an advocate for parity between Western and African epistemologies, her visit provided scholars at MSU with the opportunity to discuss the relationships among intellectual heritage, science, and colonialism. As a researcher and evaluator in the field of HIV infection, I had special interest in hearing her point of view. Many HIV scholars cite the entrenched divide between indigenous approaches to African and Western medicine as a major factor leading former South African 
President Mbeki to fail to provide antiretroviral treatment to his people, thereby contributing to the deaths of an estimated three million South Africans. I hoped she might provide me insight on how to avoid pitting knowledge systems against one another in the way that unfolded in South Africa, with its deadly consequences that still haunt the country today. Through discussion with her, I might think more precisely about how to meet my responsibilities to society as a member of a data-driven profession and do so in a culturally responsive way.

The interaction did not take the intellectual turn I expected. She and I were seated at a long meeting table, along with several graduate students. Each person in turn introduced themselves to her and described the work that they did. Last in the queue, I explained that I had devoted the bulk of my career to research and evaluation work related to HIV infection and on the health and wellbeing of gay, lesbian, bisexual, and transgender people. ${ }^{2}$ I've done a little bit of work on the harms of conversion therapy for sexual and gender minorities, which I also might have mentioned. As I completed my self-introduction, the warmth began to dissipate from her eyes. She no longer smiled, at least when she looked directly at me. She assumed a stern and disapproving countenance, openly discarding the graciousness she initially displayed. Over the course of her visit, whenever she addressed me, she made it teeth-clenched obvious that she believed those who are same-sex attracted or transgendered are not deserving of the rights of others. My perspective that there was anything normal about same-sex attraction was flatly wrong, immoral, ugly, dangerous, and corrupting, and certainly not African. I was to travel the following week as an ambassador of the American Evaluation Association (AEA) to the African Evaluation Association (AfrEA) to stay in her home country, one well known for its antagonism towards and open neglect of its sexual and gender minority citizens. Although 
I took some comfort in the fact that her beliefs defied international consensus agreements to ensure the healthy lives and wellbeing of all people of all ages, goals to which I believe evaluators ought to contribute through their work, I suddenly wondered how welcome I might be at AfrEA. The moment reminded me how fraught and difficult it can be to traverse cultures respectfully and appreciatively when deeply ingrained cultural ways of viewing the world sharply contradict my own. I was also troubled because I knew something about, though I make no claim to fully understand, the close entanglement of AIDS denialism and the movement to protect indigenous knowledge in the South African context. I worried that homophobia was similarly entangled, an entanglement that might create tremendous challenges for the evaluator who aims to work in a culturally responsive manner and whose work so often involves pursuit of the rights of sexual and gender minorities to good health.

I am unsettled, too, that culturally responsive evaluators have given so little attention to sexual orientation and gender identity and expression within diverse cultural contexts. Despite the inclusion of sexual orientation in the cultural competence statement promulgated by AEA, when I read case examples of culturally responsive evaluation projects in our books and journals, the context in which I conduct my evaluation practice is rarely described. There are no gay bars and clubs. There are no nighttime streets. There are no voguing houses. There are no sex-work strolls. There are no homeless, runaway, and drop-in centres for sexual and gender minority teenagers. There are no parks and restrooms and train stations where people search for sexual partners. The people with whom I work most closely as an evaluator and in my academic research programme-most often young Black gay and bisexual teenagers and young adults-are similarly absent in the pages of my go-to evaluation journals. There are intellectual spaces for evaluators working in these arenas, certainly, 
such as specialty journals and conferences, but these are most often places created by scholars on sexual and gender minority concerns in other disciplines such as public health and psychology. AEA's Topical Interest Group (TIG) on Lesbian, Gay, Bisexual, and Transgender issues is an important and valuable exception, but its impact on evaluation scholarship is far from fully realised or in the mainstream.

Anxious, I called Stafford, sure he had made a mistake by inviting me to CREA. Following the customary hellos and how-do-you-dos, I sought to set him straight. Disinvite me now before it is too late! That part of our conversation went something like this:

"Stafford. I'm very appreciative of your asking me to speak at CREA. But, I'm frankly a little surprised. You do know my evaluation work is, for the most part, focused on gay and bisexual folk and people living with HIV. Often both.” I paused. “That's pretty far afield of my understanding of the concerns and interests of people who come to conferences with the expectation of talking about ethnic and racial cultural issues. In my work, racial cultural issues are always positioned within GBLT issues."

Stafford interrupted me. "Right. Yes. Exactly. You answered your own question. We've left sexual orientation out. And, we shouldn't have. That's the mistake."

If that is the mistake, in this talk I have only one goal, which is to convince you that culturally responsive evaluators must start to make conscious decisions about acknowledging the presence of sexual and gender minority people in the evaluation studies that they conduct. By conscious, I mean that evaluation teams ought to explicitly consider whether and why it might be important to understand and document sexual and gender minority people's experience of programmes and policies and to be clear about the consequences and trade-offs of not collecting evaluative data that speak to their experiences. Evaluators must become attuned to the fact that in their work some of the 
programme participants, purposely or incidentally, are gay, lesbian, bisexual, and transgendered. Evaluators must become more mindful that the experience of a programme may be profoundly different for these individuals, given the extent to which the programme is attentive to their concerns and needs. With that goal in mind, let me start first by introducing myself to you not through the conventional listing of my academic credentials. I do so because my academic history, the nearly 100 articles and book chapters chronicling my work, often matters far less to my clients and the people with whom I work than my personal history and the facts of where I was raised.

In around 1970, my late parents bought a house at 114 Ocean Walk in a place named Fire Island Pines, when it had not yet become as legendary as it is today. Prior to their purchase and starting in about 1961, we had always stayed at the Osborne's home on Shady Walk not far from where open dunes containing an area known as the meat rack separate the community of Cherry Grove from the Pines. What we could afford was quite modest by comparison to the Osborne's home, but it was closer to the harbour, a distinct advantage in a household with three small children and in a community where there are no cars, transportation is your feet, and groceries are fetched from the only grocery store by toting your sacks along behind you in a Radio Flyer wagon.

Our house was among the 10 or 20 built in the Pines back during the late 1940 s or early 1950s. The house was sold to my parents cheaply because it was rundown and battered from being overstuffed with summer renters who had paid its former owner thousands of dollars just to spend a weekend or two there. The wrap-around porch sagged. The floor of the outdoor shower was coated in a thick layer of green, slimy moss. Inside was an eclectic mix of decrepit furniture picked from the refuse of the annual spring redecorating purges that occurred in the finer first-dune homes featured in Architectural Digest 
and whatever interesting debris happened to wash up on the beach. The wood floors were scuffed bare from sand. The linoleum counter was as warped as the driftwood that occasionally floated up on shore from shipwrecks and houses taken out to sea by hurricanes somewhere down the Atlantic coast.

Prior to it becoming ours, men rented out the tiny utility room housing the hot water heater under the little house. To get to this room, you had to jump off the side of the deck into the sand, inch along its side to avoid the overgrown patches of poison ivy and the beach plums that run up and over the ridge of the dune that marks the property line on the east, and then crouch under the decking and walk stooped at the waist about another 10 feet towards the underbelly of the deck. The door to the room always stood partially ajar because sand seeped underneath and around it in buckets, preventing it from closing. The room had a small window of cracked glass looking out at the underside of the deck and a patch of poison ivy, its sill littered with pellets of mouse shit. Spiders' webs adorned the low ceiling, which was far too low for a man of modest stature to stand fully upright. The hot water heater sat in the far northwest corner, creating a stifling heat in that end of the coffin-like room. There was just enough space in the room to place a camping cot beside the water heater. Summer after summer throughout the 1960s, countless young gay men had paid to sleep on that cot in that room just to be themselves.

As a child and teenaged resident of the Pines, I did many odd jobs. On Friday nights, my little sister and I waited by the dock in the harbour where the ferry boats, the Duchess and the Empress, arrived carrying men from all over the New York metropolitan region eager to leave mainland society behind for a weekend of uncloseted freedom. As the men poured off the boat, we shouted "wagon" until we each attracted a customer. We pulled our rusty wagons laden with 
luggage, groceries, and party paraphernalia along the boardwalks to our customers' summer rental homes. We made 50 cents and on rare occasions a dollar each trip for our effort. This sometimes led to other jobs, such as serving drinks at lavish parties or distributing the schedule of that season's high-camp movie showings, thumbtacking it to each house. As a preteen, I was paid to cut out paper rose petals so that they could be dropped on Holly Woodlawn, the late Puerto Rican transgender star and perennial favourite of Andy Warhol, as she made her entrance at one of the Pines' many lavish parties. I was rewarded for this some years later at about age 14 with a broken foot when Holly accidentally stepped on me during high tea at the Blue Whale. My brother once dressed up as Carmen Miranda and sold plastic fruit to men who didn't have quite enough bananas on their heads to merit entering the Carmen Miranda party. My sister and I were "adopted" for several years as the fictive sisters of two drag queens in-the-making, one of whom aspired to be Olivia Newton John and constantly sang "You're the one that I want, oo, hoo, hoo", as all 6'4" inches of her willowy frame danced about in thin black lamé pants, and the other of whom, a pint-sized 20-year-old hair stylist from Puerto Rico, gave me the dress right off her back, with a purse she made to match, the latter of which I still have. I saw the boat arrive for the very first drag Invasion of the Pines in 1976 and made sure to see it annually thereafter. By the time the Village People released their 1977 hit about the place, I had become manager of the overnight shift at the Pines Pantry ice cream stand, the Encounter, which sits in front of the water taxi stand from which people shuttle between the all-night disco in the Pines and the Ice Palace in Cherry Grove. From 7 p.m. to 9 a.m., I served blondies, scooped Häagen Dazs ice cream, twirled frozen yogurt, and made fresh juices for the closeted, the out, and the drag stars of the day. And it was there, through the cutout in the wall below the grocery store, that I had the 
perfect window on the nightmare descending on our community.

Our tiny Pines community, 500 houses occupied only from early April to November, held upwards of 30 memorial services each month in the years that marked the start of the AIDS epidemic. Men walked past 114 Ocean Walk in their prime in May and wasted and gaunt in July. In May they were enviable in their perfection. In July, the skin on their legs, torso, and face was blemished with cancerous lesions, a telltale sign of Kaposi's Sarcoma. Their skin hung slack from their frames, as they shuffled down the boardwalk, some barely strong enough to hold up their heads. And, after their memorial services, each was replaced by another man, one who would stride from the Pavilion in the waning starlight of June at 4 a.m., sweat soaked from a night of dancing and, seemingly overnight, waste away, never to return the following season. People were frightened. Friendship networks were devastated. And, when I stepped off the island each fall and headed reluctantly back to take up life on the mainland, it always struck me hard how little people outside of Fire Island or Greenwich Village or the Castro knew or cared.

I did what everyone else around me did during those years. I volunteered. I distributed condoms and safer sex information. I raised money. I went to memorial services. When Gay Men's Health Crisis (GMHC), the oldest AIDS organisation in New York City, needed help with some of the early evaluations they were conducting of their safer sex programmes, I leapt. I had just started graduate school. I possessed only the vaguest sense of what I was doing. But it didn't matter. It was a crisis. I stayed at GMHC for over 7 years, working alongside people who I knew might die in months. It was a daily part of the work. So was arriving in the morning to find I had been tasked with someone else's job because they were suddenly too unwell to do the work or had died so rapidly no one had sufficient time to prepare to fill their shoes. Craig Harris, David Austin, Benjamin McDaniel, 
Lew Kattoff, Guillermo Vasquez, Joe del Ponte. It never stopped. My basic training as an evaluator occurred less in the classroom than it did in the trenches at GMHC.

Asa Hilliard suggested that a culturally responsive evaluator can be distinguished by her display of three characteristics. He suggested she is rooted in a deep understanding of the cultural traditions of a group. She identifies with the group and is in some sense a part of it. She sees the children of the group as she would her own. Thirty-five years living on Fire Island and 7 plus years on staff at GMHC provided me a base for developing an evaluation practice that I hope still carries these hallmarks years after leaving GMHC for an academic post.

Sexual minority populations do not necessarily comprise a cohesive group, imbued with a well-articulated and shared system of values, consistent with Hilliard's use of the term. Sexual minority people are diverse in nationality, age, race, ethnicity, class, disability status, in emotional, romantic, and sexual attractions, and in gender identity and expression. There may be no obvious connections among the members of the population, particularly in settings that openly discourage their acting as an organised, cohesive community. What sexual minority people do share, however, is a unique form of social invisibility related to a defining personal characteristic, whether individuals form identities around that characteristic or not. In some country contexts, the social and legal penalties for expressing a sexual or gender minority identity are stiff. In 72 countries around the world, from Jamaica to Saudi Arabia to Chechnya, engaging in consensual same-sex sexual behaviour among males is punishable under the law, with sanctioned punishments including castration, torture, mandatory decade-long imprisonment, and death. In 45 countries, these laws apply to women as well. In 57 countries, transgender people are similarly criminalised. In settings such as these, claiming a 
sexual and gender minority identity may be conditioned by intense fear and shame. Exercising the right to ordinary opportunities may be difficult, if not impossible.

Despite a very limited effort to collect data on sexual and gender minority people globally and domestically, over the past 30 years it has become increasingly clear that when researchers and evaluators do ask, sexual and gender minority populations suffer from an array of health and mental problems at rates far higher than observed in the general population. The stress of oppression weighs heavily. Entrenched disparities, some of which have reached epidemic levels, have been documented across the many different subgroups that comprise sexual and gender minority populations, and across the domains of infectious disease, chronic noninfectious disease, psychological illness, and social pathology, most notably victimisation from multiple forms of individual and structural violence.

For instance, middle-aged and senior sexual and gender minority adults experience higher rates of social isolation and lack of supports when compared to straight and to cisgender people. They also suffer greater financial instability, due in part to lifelong experiences of employment discrimination and underemployment. Whether and how well programmes for the ageing account for the special needs and experiences of older sexual and gender minority adults is largely unknown.

Several recent studies using national health surveillance data document higher rates of multiple diseases and chronic conditions among sexual minorities including stroke, heart attack, asthma, cancer, arthritis, obesity, lung disease, diabetes, and hypertension, and high rates of health-harming behaviours that are often associated with stress, including excessive alcohol consumption and smoking. Sexual minority adults report far higher prevalence of adverse childhood experiences, including childhood sexual abuse, emotional 
abuse, and physical abuse. Across studies, roughly $22 \%$ of sexual minority males and $34 \%$ of females report sexual abuse experiences in childhood. Disaggregated by race, even higher rates are reported among males from racial minority backgrounds. Indeed, in a statewide needs assessment of Black gay and bisexual male teenagers and emerging adults I conducted for the State of Michigan some years ago, we found $33 \%$ had histories of sexual abuse, $74 \%$ of physical violence, and $91 \%$ of emotional abuse. In another evaluation, Black lesbian and bisexual girls commonly reported sexual abuse experiences, often at the hands of adult male relatives convinced that they could "convert" them to heterosexuality through rape or who used sexual violence as means to punish them for their presumed deviance-to teach them a lesson. Whether and how well systems of health and mental health care respond to these complex needs and vulnerabilities is not well known.

In too many instances, settings from churches to prisons to schools to commercial establishments engender and reinforce harm to sexual and gender minority people. Sexual minority youth face harassment, discrimination, and marginalisation in school environments at rates far greater than their heterosexual and cisgender peers, which has deleterious effects on their academic achievement and psychological wellbeing. As early as middle school, gender atypical boys experience greater levels of anxiety and social isolation and depression; their distress is magnified in schools in which gender typicality is a salient norm. How well ordinary school-based climate efforts, anti-bullying programmes, and specialised programmes aimed at healthy manhood development address the specific needs of sexual and gender minority youth is largely unknown.

Sexual minorities are disproportionately incarcerated, especially women. Recent work from Ilan Meyer and colleagues at the Williams Institute at $\mathrm{UCLA}^{3}$ suggests rates of incarceration of sexual 
minority people is roughly three times that of the US adult population. Moreover, in prison, sexual minorities are more likely to experience punitive sanctions including solitary confinement. How well programmes addressing needs of formerly incarcerated people account for the unique concerns, needs, and experiences of sexual and gender minority people is largely unknown.

Mounting evidence suggests that pervasive structural stigma against sexual and gender minority people fuels these disparities in health and wellbeing. Structural stigma constrains the opportunities that are available to achieve optimum health by allowing overt and legalised discrimination against certain groups of people, providing a normative basis for their ill treatment, and by fostering their social isolation. Structural stigmas manifest in where people can live, how they may be treated when they engage with local institutions, and how much we as a society are willing to spend on providing access to nondiscriminatory and affirming prevention and treatment. Structural stigma shapes individuals' social and material experiences through the regulation and distribution of opportunity and punishment.

In the US, anti-gay structural stigma at the community level predicts all-cause premature mortality among sexual minority adults, after accounting for multiple individual-level and confounding neighbourhood-level factors. On average, sexual minorities living in communities scoring high on a measure of gay structural stigma experience a 12-year shortened life expectancy. In addition, their deaths from conditions associated with stress (e.g., suicide, homicide, cardiac failure) occur at significantly higher rates if they live in a high-stigma community when compared to those residing in low-stigma communities and to heterosexuals living in either type of community. Studies link structural sexual minority stigma to individuals' discomfort interacting with primary care providers around discussions of sex and engagement in high-risk sexual activity. 
Structural stigma is linked to denial of healthcare services and provision of substandard care. Discrimination in the provision of health and mental health care is also associated with delaying access to care until diseases are so advanced they are likely to lead to permanent disability or premature mortality.

Striking, too, is the lack of basic investment in research, intervention development, and evaluation. Only 698 studies funded by the National Institutes of Health over a 23-year period addressed sexual and gender minority health concerns. That amounts to onetenth of a percent. Of these, the vast majority (75\%) focused on HIV, enrolling predominantly White samples. No intervention studies were funded in important health areas including maternal and infant health, exercise, physical activity, nutrition, diabetes, oral health, and cardiovascular disease. These data suggest many subgroups of sexual and gender minority people are normatively excluded from the most rigorous programmes of health-focused intervention and evaluation research in the United States.

My own evaluation practice sits within a political and social context in which AIDS stigma, sexual and gender minority stigma, and racism create a perfect storm. The photograph on the screen is from the lead article in the June 6th edition of the Sunday New York Times Magazine. Linda Villarosa, a longtime chronicler of the impact of the AIDS epidemic on Black queer communities, notes in this article that if the US Black gay male population were a country, that country would boast the highest rate of HIV in the world. The current estimates suggest that half of all Black sexual minority teenagers in the US today will acquire HIV by their 40th birthday. This 1-outof-every-2 rate contrasts against a rate of infection estimated at 1 out of 11 for a White gay man and 1 out of 99 people in the general population. In the article, Villarosa dissects the effects of being Black within the context of a largely White AIDS movement and of being 
a sexual minority within the context of a Black community that is publicly ambivalent about its sexual minority members. She writes:

Including gay Black men in the literature and understanding of the origins of the disease and its treatment could have meant earlier outreach, more of a voice and a standing in HIV/AIDS advocacy organizations, and access to the cultural and financial power of the LGBT community that would rise up to demand government action. But 35 years of neglect, compounded by poverty and inadequate local health care infrastructure, have left too many black gay and bisexual men falling through a series of safety nets. (Villarosa, 2017, para. 20)

She quotes former senior policy adviser to the Obama administration's Office of National AIDS Policy, Gregario Millett, as saying "We are going to eventually end AIDS in the United States, but I fear it's not going to happen for black MSM. We have waited too long" (Villarosa, 2017, para. 36). Cathy Cohen, a scholar of the AIDS epidemic and of Black sexual and gender minority people, notes that in the White gay and lesbian communities, AIDS became a symbol of the struggle for rights and entitlements (Cohen, 1999). Black gay and bisexual people were left on their own, marginalised within the context of both the Black and the organised White gay communities.

It is within this domestic and global context that I would argue the failure to collect and disaggregate evaluation data by sexual orientation and gender identity status becomes a matter of life and death, whether those data pertain to children in schools, elderly people in nursing homes, people accessing post-sexual assault services, ex-offenders enrolled in re-entry or job training programmes, all of it. Evidence-based policies and programmes that eliminate stigma and discrimination based on sexual orientation or gender identity and expression require it. Improving the lives of sexual and gender minority children, teens, and adults requires it. If in evaluation study 
after evaluation study, sexual and gender minority people continue to remain invisible, we will have no way to determine progress towards health equity for all. It is a problem, given the known disparities in wellbeing, that so little can be said about whether programmes and policies benefit sexual and gender minority people and to what degree. Randall Sell, a longtime public health researcher and AIDS activist, charges that by not being able to say whether Black lesbians fare the same as their heterosexual sisters or same-gender-loving ${ }^{4}$ Black men fare the same as their heterosexual brothers or Black transwomen fare the same as their cisgender sisters, we commit a form of malpractice (Sell, 2017). Global advocacy rights groups for sexual minority men and transgender people plead for evaluators' help. Interventionists working on affirmative approaches to sexual and gender minority populations want to access evaluation expertise. They know evaluative evidence allows us to discover, to build, and to influence. They know evaluative evidence steeped in the experience of others allows us to find ways to identify with those who are not exactly like us. They know evaluators bear unique responsibility to tell people's stories of policies and programmes and to document their needs. It is our charge. To satisfy that responsibility, we must recognise and see those who hide in plain sight.

I have no doubt that my context is remarkably different from yours. However, I think that the general principles I use to guide my evaluation work have broad applicability for stepping us towards a culturally responsive practice that gives visibility to sexual and gender minority citizens within the diverse cultural and programme spaces within which we each work.

Inclusion. Working under the imperatives of funders is always difficult. Too often we are under pressure to move quickly with too few resources. To just get the work done. But, doing so puts us in danger of replicating stigma, disempowerment, and inequality by giving the 
roles of control and engagement short shrift. The most fundamental lesson of my time at GMHC has been to ground my evaluation work in the ethic of inclusion and to argue for its importance in time, in resources, and in power. Allowing gay and bisexual men to take leadership within the context of the evaluation projects I take on and doing so in ways that acknowledge and place trust in their abilities is essential to reducing inequality and creating opportunities for their individual expression and voice. In the needs assessment study that I mentioned earlier, in which we found high rates of exposure to multiple forms of violence, I intentionally structured the project to share power with young Black gay and bisexual men throughout the state of Michigan. The study was commissioned by the State Department of Health through its US Centers for Disease Control and Prevention (CDC) - and US Health Resources and Services Administration (HRSA)-mandated community advisory board. The effort represented the first time the state had ever sought to systematically understand its young Black gay and bisexual male citizens. Regrettably, the study was driven by a sense of impending panic, as the state was saddled with dramatic increases in new infections in young Black gay men over the 6 years prior to the study, along with a syphilis epidemic, which is unusual in a population so young. Rather than assemble an advisory board, I hired six young men under age 24 who lived in the highest sero-prevalence counties in Michigan to serve as the study co-investigators. I budgeted for them to be compensated for their time and effort, for their transportation to and from meetings, and for meals that we shared. I arranged and paid for drivers for those who lacked access to their own transportation. We made all decisions as a team. The young men worked with me on determining how to address the informational needs identified by our stakeholders while also making sure that we covered important content that would not occur to the adult stakeholders and study 
commissioners. We spent a day together sorting through what stakeholders wanted to know, based on key informant interviews with them. At one point, one of the young men looked up from the table and said, "None of this is about us. It's all about them. What do you mean, where can young men be found? We're in school. We're in the grocery store. We're at church. Shit. Ask that shit when you are doing your evaluation of your services. This study is ours. It's supposed to be about us." The young men promptly inserted questions on family, church, mentorship, life in the gay community as a young Black man, and violence.

They chose when we should use quantitative instruments and when we should ask men to tell their stories in an open-ended fashion. They selected the recruitment strategies and venues. They screened prospective interviewers and trained them. They participated in decisions to retain some interviewers and let others go after a trial period. They assisted in recruitment. They shaped procedures to give prospective interviewees the choice of whether they wanted to be interviewed by a man or a woman, a person of a particular racial or ethnic background, or a person who was identified as gay, lesbian, or bisexual. They helped us process the results. They co-presented the results to a convened meeting of the statewide council and held their own town hall meeting with youth on the results in Detroit. They shaped recommendations. They read and approved all reports and academic articles based on the work. We shared it.

In the data that we collected to evaluate our own evaluation, we see evidence of the extent to which their inclusion created a climate that felt safe and validating to respondents and empowering to the co-investigators:

The survey was cool. We actually get to express a piece of [us] with someone who actually wants to hear it. 
This is great. I feel like I'm like a part of something. Everyone wants to change the world, but no one ever does anything. Maybe I've helped bring us one step closer. Who knows? (Needs Assessment respondents)

Determining what questions to ask or take out was all based on my personal view. I was able to just give my true feeling of what is best for myself and my community. (Co-investigator)

We also found evidence that their inclusion and the prominent role they played in the work facilitated influence:

I think engaging the young men throughout the process, including having them participate in presentation of data, enhanced the credibility with internal as well as external partners. I have also found that people seem to be paying more attention to this study-certainly the content is urgent-but also perhaps because it felt from/ with the community. (Health Department representative)

In my opinion, engaging the young men in the manner in which you did was absolutely essential to the evaluation process and greatly strengthened the endeavor. It gave the research a huge amount of credibility. (Health Department representative)

I think that providers paid more attention to the findings because they saw that YBMSM were involved in every step of the process. I've had more conversations with agencies about the study and how it informs their work than I have about the past assessments that we've funded. (Health Department project officer)

Young men's inclusion prompted the state's planning council to question whether its focus on a limited cadre of individually focused interventions promoting HIV counselling and testing was best, versus strategies integrating mental health, violence recovery, and sexual health; family-acceptance work and support building; and stigma reduction. Young men helped these decision makers to see more 
clearly how their wellbeing was constrained by inattention to viewing them holistically and as members of the larger communities in which they lived, worked, and attended school.

Affirmation. In a recent article in New Directions for Evaluation, Fred Carden (2017) notes that evaluating with a concern for equity means getting into the specifics and understanding processes. I agree. I think it is especially important in the case of sexual and gender minority people to evaluate with an eye towards generating understanding of what constitutes a culturally specific, affirming and nonstigmatising programme, one which supports resilience. I evaluated the implementation of one of the few evidence-based programmes in the CDC compendium designed for young gay men called the Mpowerment Project. The replication programme I evaluated was housed in downtown Detroit in a loft apartment perched two floors above a pizza shop.

The Mpowerment Project was originally designed by a research team in San Francisco at the University of California and tested in Eugene, Oregon and Santa Barbara, California. Although the programme has since undergone extensive testing with gay men in countries from Peru to South Africa to Lebanon and in multiple racial-ethnic minority groups in the US, at the time I conducted the evaluation, very little was known about the challenges an organisation might face in carrying out the programme or about its appropriate adaptation to local ecologies that differed in their racial, geographic, and socioeconomic characteristics from the programme's origin communities. Unlike the original trial programme that enrolled a largely White middle-class group of young men and occurred in small college towns, the Detroit effort was implemented to create a prevention infrastructure exclusively for Detroit's urban Black gay and bisexual male youth aged 13 to 24 . The evaluation occurred during the programme's third year of implementation and centred 
on understanding the adaptations staff made to fit life in Detroit for Black sexual and gender minority young people. The evaluation design we used was ethnographic and relied on qualitative interviews with staff and programme participants, a comprehensive review of all programme records and archives, and 112 hours of observations carried out over a period of roughly 15 months.

The changes the staff made were illuminating. Staff believed the original programme reduced men to one dimension-their gay identity_rather than foster their holistic development. As young Black men who must face the prejudices and challenges of navigating through racism and homophobia, helping young men to develop a holistic and positive self-perception was of cardinal importance. Issues related to promoting academic and career success, disclosure, homelessness, juvenile justice, and poverty took precedence. The programme became about much more than HIV. Black identities, experiences, and concerns were hard to locate in the programme's original trainings, scripts, exercises, activities, and assumptions about the organisation of community life and local resources. Staff made major alterations to the programme's leadership structure, staffing model, and core activities. As the men refashioned the programme, they drew on familiar cultural institutions for models, such as Pan Hellenic fraternities. ${ }^{5}$ Leadership development took on a highly prominent role. The programme functioned as a fictive family for young men. Every night, the executive director made a meal for the young men who might be there to attend a discussion or just to hang out in a safe space. The young men gathered around the kitchen counter, hands clasped, heads bowed, before breaking bread together. Women were welcome in the space, reflecting the importance of mothers, sisters, aunts, and grandmothers in young men's lives, and were also part of meals and activities. By elucidating the elements of a culturally grounded affirmative programme recreated 
by and for young Black gay, bisexual, and transgender men, we were able to obtain a rich sense of why young men on the other side of the state piled into cars and drove over 150 miles one way simply to have a meal or meet with a mentor among the young men in leadership roles or watch television in the space.

Safety. The final guiding principle I'd like to highlight concerns safety. Participation in evaluation studies and accessing services can be a dangerous enterprise in some settings. Even when it is not dangerous, it may feel so. Sexual and gender minority people must retain control over their safety and to preserve it, their privacy. I take extra precaution to design procedures that are mindful of the stigmatised position of these individuals and their concerns about matters such as inadvertent disclosures of their sexual orientation, gender identity, HIV status, drug use, sex-work history, or other personal characteristics and experiences to family, to friends, to law enforcement, or to providers whose knowledge of any of these things is nonessential. In its simplest form, I try to combine the ambition to protect individuals' dignity with the goal of ensuring their physical and emotional safety. The procedures in every project are somewhat different, but I invest substantial time in thinking and talking these through with programme staff and the intended beneficiaries of their efforts. My team and I are especially sensitive to the fact we must protect people from being "outed" about sexual orientation or HIV status by our study materials, recruitment and data collection procedures, and reporting. Nothing in how we set up our studies should permit inadvertent disclosure. For instance, at MSU we are required to provide specific destination addresses to receive reimbursement for automobile mileage. We routinely secure permission to use nearest cross-streets so that specific residences cannot be identified, as our evaluations are obviously of people with HIV or people who are sexual minority or both and we had no control over the storage or 
destruction of reimbursement records. We seek waivers of documentation of informed consent and of parental consent as a matter of routine to maximise the protection of individuals' identities. We avoid identifiable data collection, if possible, but go the extra mile to put in place rigorous physical and electronic data security procedures, including procedures pertinent to record abstraction, transportation, storage, and destruction.

We make similar effort around psychological safety in data collection, letting those we interact with face to face choose what is safe for them. We develop procedures to address the range of issues and challenges sexual and gender minority people are likely to report. We encounter individuals who are engaging in unprotected sex. We encounter individuals who have not disclosed their HIV status to their sexual partners, to their primary care physicians, or to their parents. We encounter individuals who are having difficulty complying with medication regimens. We encounter individuals who are extremely ill and have fallen out of care. We encounter people who would not seek prevention, testing, or care locally out of fear of being outed. My staff are highly trained in the target population and in HIV. We develop procedures to address these and other possibilities. I think what is important here is less what we might do in any specific instance than the notion we carry into each study that our evaluation should not endanger or stigmatise and that we should be planful about how best we can develop procedures that preserve and promote individuals' dignity.

My ask of you is, on its face, simple. Help ensure that the programmes and policies you work with every day are enfranchising and increase the life opportunities of sexual and gender minority people. You need not go so far as to change your practice to be like me, an evaluator who limits herself to the concerns of people living with or at high risk of exposure to HIV and to sexual and gender minority 
concerns. Yet, think about what you might add to the base of knowledge on sexual and gender minority people by obtaining information on sexual orientation and gender identity in the programmes you evaluate. Think of the potential you have in your studies to understand the practices that shape diverse people's experiences and to highlight practices that pit one identity against another or define what we consider normal in ways that are harmful. What might happen if, whenever it was possible, you posed the question "How might this programme or policy be experienced by and impact on a person who is a sexual minority or transgendered?" As a community of culturally responsive evaluators, we share the ideals of diversity and inclusion. We believe it is the paramount symbol of living in a just world, a world that we are far from having attained. If we are to attain the social justice we seek and to conduct our evaluation work to best support these ideals, we must continue to apply our skills to the problems of diversity and inclusion in ways that make visible the communities of people who are in plain sight.

\section{Notes}

1 Professor Stafford Hood, Head of Centre for Culturally Responsive Evaluation and Assessment (CREA).

2 In this talk, I use "sexual minority" as an umbrella term to refer to all individuals whose sexual attractions include people of their same gender. I similarly use "gender minority" to refer to those whose gender identity differs from the sex that they were assigned at birth or whose gender expression does not conform to dominant cultural norms. I use the terms "lesbian", "gay", "bisexual”, and "transgender", or the acronym "LGBT", when referring to those sexual and gender minority people who express these identities. I typically use these more specific terms when they best characterise study samples or the focus of a piece of scholarship. I quote people who use the term "men who have sex with men" or "MSM" to refer to men who are same-gender attracted and who do not necessarily identify as gay or bisexual. In retrospect, I should have changed the title of my talk, which I had to supply to CREA months before I put pen to paper, to better reflect its final content and broad emphasis on sexual and gender minority people. 
3 University of California at Los Angeles.

4 Same-gender-loving is a common expression among Black Americans to refer to men who are same-gender attracted.

5 The National Pan Hellenic Fraternities comprise nine historically Black Greek letter fraternities and sororities, each of which emphasise academic excellence and service to the Black community.

\section{References}

Aggleton, P., \& Parker, R. (2015). Moving beyond biomedicalization in the HIV response: Implications for community involvement and community leadership among men who have sex with men and transgender people. American Journal of Public Health, 105(8), 1552-1558.

Andrasick, M. P., Chandler, C. P., Wakefield, S., Kripke, K., \& Eckstein, D. (2014). Bridging the divide: HIV prevention research and Black men who have sex with men. American Journal of Public Health, 104(4), 708-714.

APA Task Force on Appropriate Therapeutic Responses to Sexual Orientation. (2009). Report of the Task Force on Appropriate Therapeutic Responses to Sexual Orientation. Washington, DC: American Psychological Association.

Austin, A., Herrick, H., \& Proescholdbell, S. (2016). Adverse childhood experiences related to poor adult health among lesbian, gay, and bisexual individuals. American Journal of Public Health, 106(2), 314-320.

Carden, F. (2017). Building evaluation capacity to address problems of equity. New Directions for Evaluation, No. 154, 115-125.

Cohen, C. J. (1999). The boundaries of Blackness: AIDS and the breakdown of Black politics. Chicago: University of Chicago Press.

Committee on Lesbian, Gay, Bisexual, and Transgender Health Issues and Research Gaps and Opportunities; Board on the Health of Select Populations; Institute of Medicine. (2011). Introduction. In The health of lesbian, gay, bisexual, and transgender people: Building a foundation for better understanding (pp. 89-140). Washington, DC: National Academies Press. 
Coulter, R. W., Kenst, K. S., Bowen, D. J., \& Scout. (2014). Research funded by the National Institutes of Health on the health of lesbian, gay, bisexual, and transgender populations. American Journal of Public Health, e105-e112.

Durso, L. E. (2017). Data move us closer to full equality by speaking for those who cannot: Advocating for LGBT older adults. American Journal of Public Health, 107(8), 1219-1220.

Epstein, S. (1994). Impure science: AIDS, activism, and the politics of knowledge. Berkley: University of California Press.

France, D. (2016). How to survive a plague: The inside story of how citizens and science tamed AIDS. New York: Alfred A. Knopf.

Fredriksen-Goldsen, K. I., Kim, H. J., Shui, C., \& Bryan, A. E. B. (2017). Chronic health conditions and key health indicators among lesbian, gay, and bisexual older US adults, 2013-2014. American Journal of Public Health, 107(8), 1332-1338.

Green, L. J. F. (2012). Beyond South Africa's “indigenous knowledgescience" wars. South African Journal of Science, 108(7/8).

Greenlee, K. (2017). Why I am opposed to the elimination of the sexual orientation question by the administration for community living. American Journal of Public Health, 107(8), 1211-1212.

Hatzenbuehler, M. L., Bellatorre, A., Lee, Y., Finch, B., Muennig, P., \& Fiscella, K. (2014). Structural stigma and all-cause mortality in sexual minority populations. Social Science and Medicine, 103, 33-41.

Hughes, A. K., Harold, R. D., \& Boyer, J. M. (2011). Awareness of LGBT issues among aging services network providers. Journal of Gerontological Social Work, 54(7), 659-677.

Hughto, J. M., White, G. R., Murchison, K. C., Pachankis, J. E., \& Reisner, S. L. (2016). Geographic and individual differences in healthcare access for U.S. transgender adults: A multilevel analysis. LGBT Health, 3, 424-433. 
International Lesbian, Gay, Bisexual, Trans, and Intersex Association. (2017). State-sponsored homophobia report, 12th Edition, May 2017. Geneva: Author.

Koblin, L. (2012). Disparities in HIV/AIDS in black men who have sex with men. The Lancet, 380(9839), 316-318.

Kosciw, J. G., Palmer, H. A., \& Kull, R. M. (2015). Reflecting resiliency: Openness about sexual orientation and/or gender identity and its relationship to well-being and educational outcomes for LGBT students. American Journal of Community Psychology, 55(1-2), 167-178.

Krehely, J. (2009, December 21). How to close the LGBT health disparities gap. Retrieved 26 December 2016, from Center for American Progress: https://www.americanprogress.org/issues/lgbt/reports/2009/12/21/7048/ how-to-close-the-lgbt-health-disparities-gap/

Lamda Legal. (2010). When health care isn't caring: Lambda Legal's survey of discrimination against LGBT people and people with HIV. New York: Author.

Loewy, K. L. (2017). Erasing LGBT people from federal data collection: A need for vigilance. American Journal of Public Health, 107(8), 1217-1218.

Matthews, D. D., Smith, J. C., Brown, A. L., \& Malebranche, D. J. (2016). Reconciling epidemiology and social justice in the public health discourse around the sexual networks of Black men who have sex with men. Perspectives for the Social Sciences, 106(5), 808-814.

Meyer, I. H., Flores, A. R., Stemple, L., Romero, A. P., Wilson, B. D. M., \& Herman, J. L. (2017). Incarceration rates and traits of sexual minorities in the United States: National inmate survey, 2011-2012. American Journal of Public Health, 107(2), 267-273.

Miller. R. L. (2018). Evaluating HIV practices and evidence-supported programs in AIDS community-based organizations. In R. Hopson \& F. Cram (Eds.), Tackling wicked problems in complex ecologies: The role of evaluation (pp. 69-102). Stanford: Stanford University Press. 
Miller, R. L., Forney, J. C., Hubbard, P. K., \& Camacho, L. (2012). Reinventing Mpowerment for Black men: Long-term community implementation of an evidence-based program. American Journal of Community Psychology, 49, 199-214.

Miller, R. L., Janulis, P., Reed, S. J., Harper, G. W., Boyer, C. B., Ellen, $\mathrm{J}$. and the Adolescent Medicine Trials Network for HIV/AIDS Interventions. (2016). Creating youth-supportive communities: Outcomes from the Connect-to-Protect ${ }^{\circledR}(\mathrm{C} 2 \mathrm{P})$ structural change approach to youth HIV prevention. Journal of Youth and Adolescence, 45(2), 301-315.

Miller, R. L., Klotz, D., \& Eckholdt, H. M. (1998). HIV prevention with male prostitutes and patrons of hustler bars: Replication of an HIV prevention intervention. American Journal of Community Psychology, 26, 97-131.

Miller, R. L., Reed, S. J., Francisco, V., \& Ellen, J. (2012). Conflict transformation, stigma, and HIV-preventive structural change. American Journal of Community Psychology, 49, 378-392.

Miller, R. L., Reed, S. J., McNall, M., \& Forney, J. C. (2013). The effect of trauma on recent inconsistent condom use among young Black gay and bisexual men. Journal of HIVIAIDS and Social Services, 12(3-4), 349-367.

Millett, G. A., Jeffries, W. L., Peterson, J. L., Malebranche, D. J., Lane, T. Flores, S. A.....Heilig, C. M. (2012). Common roots: A contextual review of HIV epidemics in Black men who have sex with men across the African diaspora. The Lancet, 380(9839), 411-423.

Millett, G. A., Peterson, J. L., Flores, S. A., Hart, T. A., Jeffries, W. L., Wilson, P. A....Remis, R. S. (2012). Comparisons of disparities and risks of HIV infection in Black and other men who have sex with men in Canada, UK, and USA: A meta-analysis. The Lancet, 380(9839), 341-348.

MSMGF \& Outright Action International. (2017). Agenda 2030 for LGBTI health and well-being. Oakland: Author. 
Mustanski, B., Andrews, R., \& Puckett, J. A. (2016). The effects of cumulative victimization on mental health among lesbian, gay, bisexual, and transgender adolescents and young adults. American Journal of Public Health, 106(3), 527-533.

Nieves-Lugo, K., Rohrbeck, C. A., Nakamura, N., \& Zea, M. C. (2016). Interventions with lesbian, gay, bisexual, transgender, and questioning communities. In M. A. Bond, I. Serrano-Garcia, \& C. B. Keys, APA handbook of community psychology, Volume 2: Methods for community research and action for diverse groups and issues (pp. 555-569). Washington: American Psychological Association.

Patton, C. (1986). Sex and germs: The politics of AIDS. Montreal: Black Rose Books.

Patton, C. (1990). Inventing AIDS. New York: Routledge.

Prilleltensky, I. (2010). Child wellness and social inclusion: Values for action. American Journal of Community Psychology, 46(1-2), 238-249.

Prilleltensky, I. (2012). Wellness as fairness. American Journal of Community Psychology, 49(1-2), 1-21.

Reed, S. J., \& Miller, R. L. (2011). Identity and agency: The meaning and value of pregnancy for young Black lesbians. Psychology of Women Quarterly, 35(4), 571-581.

Reed, S. J., \& Miller, R. L. (2016). Thriving and adapting: Resilience, sense of community and syndemics among young Black gay and bisexual men. American Journal of Community Psychology, 57(1-2), 129-143.

Reed, S. J., Miller, R. L., Valenti, M. T., \& Timm, T. M. (2011). Baby's daddies, dick dykes, and good gay females: Black lesbian community norms and the acceptability of pregnancy. Culture, Health, and Sexuality, 13, 751-765.

Sekoni, A. O., Jolly, K., Gale, N. K., Ifaniyi, O. A., Somefun, E. O., Agaba, E. I., \& Fakayode, V. A. (2016). Provision of services to men who have sex with men in Nigeria: Students' attitudes following the passage of the same-sex marriage prohibition law. LGBT Health, 3(4), 300-307. 
Sell, R. L. (2017). LGBT health surveillance: Data = power. American Journal of Public Health, 107(6), 843-844.

Singer, M. (1994). AIDS and the health crisis of the US urban poor: The perspective of critical medical anthropology. Social Science and Medicine, 39(7), 931-948.

Stokes, J. P., Miller, R. L., \& Mundhenk, R. (1998). Toward an understanding of behaviorally bisexual men: The influence of context and culture. Canadian Journal of Human Sexuality, 7(2), 101-113.

Substance Abuse and Mental Health Services Administration. (2015). Ending conversion therapy: Supporting and affirming LGBTQ youth. Rockville, MD: Author.

Timm, T., Reed, S. J., Miller, R. L., \& Valenti, M. T. (2013). Sexual debut of young Black women who have sex with women: Implications for STI/HIV risk. Youth and Society, 45(2), 167-183.

UNAIDS. (2014). The Gap report 2014. Geneva: Author.

Villarosa, L. (2017). America's hidden HIV epidemic: Why do America's Black gay and bisexual men have a higher HIV rate than any country in the world? New York Times Magazine, 6 June 2017. Retrieved from https:// www.nytimes.com/2017/06/06/magazine/americas-hidden-hiv-epidemic. html

Wilson, B. D. M., \& Miller, R. L. (2003). Integrating culture into HIV prevention research: A review. AIDS Education and Prevention, 15(4), 184-202.

Wilson, B. D. M., \& Miller, R. L. (2002). Strategies for coping with sexual orientation among African American men who have sex with men. Journal of Black Psychology, 28, 371-391.

Wilson, C. K., West, L., Stepleman, L., Villarosa, M., Ange, B., Decker, M., \& Waller, J. L. (2014). Attitudes toward LGBT patients among students in the health professions: Influence of demographics and discipline. $L G B T$ Health, 1(3), 204-211. 


\section{The author}

Robin Lin Miller, PhD, is Professor and Chair of the Doctoral Training Program Ecological-Community Psychology and Co-Director of the Masters Training Program and Certificate in Program Evaluation at Michigan State University.

Email:mill1493@msu.edu 